\title{
A Generic Framework for Reasoning About Dynamic Networks of Infinite-State Processes
}

\author{
Ahmed Bouajjani, Yan Jurski, and Mihaela Sighireanu \\ LIAFA, University of Paris 7, Case 7014, 2 place Jussieu, 75251 Paris 05, France \\ \{abou, jurski, sighirea\}@liafa.jussieu.fr
}

\begin{abstract}
We propose a framework for reasoning about unbounded dynamic networks of infinite-state processes. We propose Constrained Petri Nets (CPN) as generic models for these networks. They can be seen as Petri nets where tokens (representing occurrences of processes) are colored by values over some potentially infinite data domain such as integers, reals, etc. Furthermore, we define a logic, called CML (colored markings logic), for the description of CPN configurations. $C M L$ is a first-order logic over tokens allowing to reason about their locations and their colors. Both CPNs and CML are parametrized by a color logic allowing to express constraints on the colors (data) associated with tokens.

We investigate the decidability of the satisfiability problem of CML and its applications in the verification of CPNs. We identify a fragment of CML for which the satisfiability problem is decidable (whenever it is the case for the underlying color logic), and which is closed under the computations of post and pre images for CPNs. These results can be used for several kinds of analysis such as invariance checking, pre-post condition reasoning, and bounded reachability analysis.
\end{abstract}

\section{Introduction}

The verification of software systems requires in general the consideration of infinitestate models. The sources of infinity in software models are multiple. One of them is the manipulation of variables and data structures ranging over infinite domains (such as integers, reals, arrays, etc). Another source of infinity is the fact that the number of processes running in parallel in the system can be either a parameter (fixed but arbitrarily large), or it can be dynamically changing due to process creation. While the verification of parameterized systems requires reasoning uniformly about the infinite family of (static) networks corresponding to any possible number of processes, the verification of dynamic systems requires reasoning about the infinite number of all possible dynamically changing network configurations.

There are many works and several approaches on the verification of infinite-state systems taking into account either the aspects related to infinite data domains, or the aspects related to unbounded network structures due to parameterization or dynamism. Concerning systems with data manipulation, a lot of work has been devoted to the verification of, for instance, finite-structure systems with unbounded counters, clocks, stacks, queues, etc. (see, e.g., [1|11|30 7 5|27|26]). On the other hand, a lot of work has been done for the verification of parameterized and dynamic networks of boolean (or finite-data domain) processes, proposing either exact model-checking and reachability 
analysis techniques for specific classes of systems (such as broadcast protocols, multithreaded programs, etc) [24|25|22|16 15], or generic algorithmic techniques (which can be approximate, or not guaranteed to terminate) such as network invariants-based approaches [31|20], and (abstract) regular model checking [13 17|3 12]. However, only few works consider both infinite data manipulation and parametric/dynamic network structures (see the paragraph on related work).

In this paper, we propose a generic framework for reasoning about parameterized and dynamic networks of concurrent processes which can manipulate (local and global) variables over infinite data domains. Our framework is parameterized by a data domain and a first-order theory on it (e.g., Presburger arithmetics on natural numbers). It consists of (1) expressive models allowing to cover a wide class of systems, and (2) a logic allowing to specify and to reason about the configurations of these models.

The models we propose are called Constrained Petri Nets (CPN for short). They are based on (place/transition) Petri nets where tokens are colored by data values. Intuitively, tokens represent different occurrences of processes, and places are associated with control locations and contain tokens corresponding to processes which are at a same control location. Since processes can manipulate local variables, each token (process occurrence) has several colors corresponding to the values of these variables. Then, configurations of our models are markings where each place contains a set of colored tokens, and transitions modify the markings as usual by removing tokens from some places and creating new ones in some other places. Transitions are guarded by constraints on the colors of tokens before and after firing the transition. We show that CPNs allow to model various aspects such as unbounded dynamic creation of processes, manipulation of local and global variables over unbounded domains such as integers, synchronization, communication through shared variables, locks, etc.

The logic we propose for specifying configurations of CPN's is called Colored Markings Logic (CML for short). It is a first order logic over tokens and their colors. It allows to reason about the presence of tokens in places, and also about the relations between the colors of these tokens. The logic CML is parametrized by a first order logic over the color domain allowing to express constraints on tokens.

We investigate the decidability of the satisfiability problem of CML and its applications in verification of CPNs. While the logic is decidable for finite color domains (such as booleans), we show that, unfortunately, the satisfiability problem of this logic becomes undecidable as soon as we consider as a color domain the set of natural numbers with the usual ordering relation (and without any arithmetical operations). We prove that this undecidability result holds already for the fragment $\forall^{*} \exists^{*}$ of the logic (in the alternation hierarchy of the quantifiers over token variables) with this color domain.

On the other hand, we prove that the satisfiability problem is decidable for the fragment $\exists * \forall^{*}$ of CML whenever the underlying color logic has a decidable satisfiability problem, e.g., Presburger arithmetics, the first-order logic of addition and multiplication over reals, etc. Moreover, we prove that the fragment $\exists^{*} \forall^{*}$ of CML is effectively closed under post and pre image computations (i.e., computation of immediate successors and immediate predecessors) for CPN's where all transition guards are also in $\exists * \forall^{*}$. We show also that the same closure results hold when we consider the fragment $\exists^{*}$ instead of $\exists^{*} \forall^{*}$. 
These generic decidability and closure results can be applied in the verification of CPN models following different approaches such as pre-post condition (Hoare triples based) reasoning, bounded reachability analysis, and inductive invariant checking. More precisely, we derive from our results mentioned above that (1) checking whether starting from a $\exists^{*} \forall^{*}$ pre-condition, a $\forall^{*} \exists^{*}$ condition holds after the execution of a transition is decidable, that (2) the bounded reachability problem between two $\exists^{*} \forall^{*}$ definable sets is decidable, and that (3) checking whether a formula defines an inductive invariant is decidable for boolean combinations of $\exists^{*}$ formulas.

These results can be used to deal with non trivial examples of systems. Indeed, in many cases, program invariants and the assertions needed to establish them fall in the considered fragments of our logic. We illustrate this by carrying out in our framework the parametric verification of a Reader-Writer lock with an arbitrarily large number of processes. This case study was introduced in [28] where the authors provide a correctness proof for the case of one reader and one writer.

For lack of space, proofs as well as the exposition of the Reader-Writer case study are omitted in this short version of the paper. They are provided in the full paper [14].

Related work: The use of unbounded Petri nets as models for parametrized networks of processes has been proposed in many existing works such as [29 24 22]. However, these works consider networks of finite-state processes and do not address the issue of manipulating infinite data domains. The extension of this idea to networks of infinitestate processes has been addressed only in very few works [4|21|18 2]. In [4], Abdulla and Jonsson consider the case of networks of 1-clock timed systems and show, using the theory of well-structured systems and well quasi orderings [127], that the verification problem for a class of safety properties is decidable. Their approach has been extended in [21 18] to a particular class of multiset rewrite systems with constraints (see also [2] for recent developments of this approach). Our modeling framework is actually inspired by these works. However, while they address the issue of deciding the verification problem of safety properties (by reduction to the coverability problem) for specific classes of systems, we consider in our work a general framework, allowing to deal in a generic way with various classes of systems, where the user can express assertions about the configurations of the system, and check automatically that they hold (using post-pre reasoning and inductive invariant checking) or that they do not hold (using bounded reachability analysis). Our framework allows to reason automatically about systems which are beyond the scoop of the techniques proposed in [4|21|182] (such as, for instance, the parametrized Reader-Writer lock system [14]).

In a series of papers, Pnueli et al. developed an approach for the verification of parameterized systems combining abstraction and proof techniques (see, e.g., [6]). This is probably one of the most advanced existing approaches allowing to deal with unbounded networks of infinite-state processes. We propose here a different framework for reasoning about these systems. In [6], the authors consider a logic on (parametricbound) arrays of integers, and they identify a fragment of this logic for which the satisfiability problem is decidable. In this fragment, they restrict the shape of the formula (quantification over indices) to formulas in the fragment $\exists^{*} \forall^{*}$ similarly to what we do, and also the class of used arithmetical constraints on indices and on the associated values. In a recent work by Bradley and al. [19], the satisfiability problem of the logic of 
unbounded arrays with integers is investigated and the authors provide a new decidable fragment, which is incomparable to the one defined in [6], but again which imposes similar restrictions on the quantification alternation in the formulas, and on the kind of constraints that can be used. In contrast with these works, we consider a logic on multisets of elements with any kind of associated data values, provided that the used theory on the data domain is decidable. For instance, we can use in our logic general Presburger constraints whereas [6] and [19] allow limited classes of constraints. On the other hand, we cannot specify faithfully unbounded arrays in our decidable fragment because formulas of the form $\forall \exists$ are needed to express that every non extremal element has a successor/predecessor. Nevertheless, for the verification of safety properties and invariant checking, expressing this fact is not necessary, and therefore, it is possible to handle in our framework all usual examples of parametrized systems (such as mutual exclusion protocols) considered in the works mentioned above.

Let us finally mention that there are recent works on logics (first-order logics, or temporal logics) over finite/infinite structures (words or trees) over infinite alphabets (which can be considered as abstract infinite data domains) [9 8[23]. The obtained positive results so far concern logics with very limited data domain (basically infinite sets with only equality, or sometimes with an ordering relation), and are based on reduction to complex problems such as reachability in Petri nets.

\section{Colored Markings Logic}

\subsection{Preliminaries}

Consider an enumerable set of tokens and let us identify this set with the set of natural numbers $\mathbb{N}$. Intuitively, tokens represent occurrences of (parallel) processes. We assume that tokens may have colors corresponding for instance to data values attached to the corresponding processes. Let $\mathbb{C}$ be a (potentially infinite) token color domain. Examples of color domains are the set of natural numbers $\mathbb{N}$ and the set of real numbers $\mathbb{R}$.

Colors are associated with tokens through coloring functions. Let $\Gamma$ be a finite set of token coloring symbols. Each element in $\Gamma$ is interpreted as a mapping from $\mathbb{N}$ (the set of tokens) to $\mathbb{C}$ (the set of colors). Then, let a valuation of the token coloring symbols be a mapping in $[\Gamma \rightarrow(\mathbb{N} \rightarrow \mathbb{C})]$.

To express constraints on token colors, we use first-order logics over the considered color domains. In the sequel we refer to such logics as color logics. Presburger arithmetics $P A=(\mathbb{N},\{0,1,+\},\{\leq\})$ is an example of such a logic. It is well known that the satisfiability problem of Presburger arithmetics is decidable. An interesting sublogic of $\mathrm{PA}$ is the difference logic $\mathrm{DL}=\left(\mathbb{N},\{0\},\left\{\leq_{k}: k \geq 0\right\}\right)$ where, for every $u, v, k \in \mathbb{N}$, $u \leq_{k} v$ holds if and only if $u-v \leq k$. The order logic on natural numbers is the sublogic of $\mathrm{DL}$ defined by $\mathrm{OL}=(\mathbb{N},\{0\}, \leq)$. Another example of a decidable logic which can be used as a color logic is the first-order theory of reals $\mathrm{FO}_{\mathbb{R}}=(\mathbb{R},\{0,1,+, \times\},\{\leq\})$.

We consider that tokens can be located at places. Let $\mathbb{P}$ be a finite set of such places. A marking is a mapping in $[\mathbb{N} \rightarrow \mathbb{P} \cup\{\perp\}]$ which associates with each token the unique place where it is located if it is defined, or $\perp$ otherwise. A colored markingis a pair $\langle M, \mu\rangle$ where $M$ is a marking and $\mu$ is a valuation of the token coloring symbols. 


\subsection{Syntax and Semantics of CML}

We define hereafter the syntax of the logic colored markings logic $\operatorname{CML}(L, \Gamma, \mathbb{P})$ which is parametrized with a color logic $L$, a finite set of token coloring symbols $\Gamma$, and a finite set of places $\mathbb{P}$. Then, let $L=(\mathbb{C}, \Omega, \Xi)$ be the first-order logic over the color domain $\mathbb{C}$ of the set of functions $\Omega$ and the set of relations $\Xi$. In the sequel, we omit all or some of the parameters of CML when their specification is not necessary.

Let $T$ be set of token variables and let $C$ be set of color variables, and assume that $T \cap C=\emptyset$. The set of CML terms (called token color terms) is given by the grammar:

$$
t::=z|\gamma(x)| \omega\left(t_{1}, \ldots, t_{n}\right)
$$

where $z \in C, \gamma \in \Gamma, x \in T$, and $\omega \in \Omega$. Then, the set of CML formulas is given by:

$$
\varphi::=x=y|p(x)| \xi\left(t_{1}, \ldots, t_{m}\right)|\neg \varphi| \varphi \vee \varphi|\exists z . \varphi| \exists x . \varphi
$$

where $x, y \in T, z \in C, p \in \mathbb{P} \cup\{\perp\}, \xi \in \Xi$, and $t_{1}, \ldots, t_{m}$ are token color terms. Boolean connectives such as conjunction $(\wedge)$ and implication $(\Rightarrow)$, and universal quantification $(\forall)$ can be defined in terms of $\neg, \vee$, and $\exists$. We also use $\exists x \in p . \varphi$ (resp. $\forall x \in p . \varphi$ ) as an abbreviation of the formula $\exists x \cdot p(x) \wedge \varphi$ (resp. $\forall x \cdot p(x) \Rightarrow \varphi$ ). Notice that the set of terms (resp. formulas) of $L$ is included in the set of terms (resp. formulas) of $\operatorname{CML}(L)$.

The notions of free/bound occurrences of variables in formulas and the notions of closed/open formulas are defined as usual in first-order logics. In the sequel, we assume w.l.o.g. that in every formula, each variable is quantified at most once.

We define a satisfaction relation between colored markings and CML formulas. For that, we need first to define the semantics of CML terms. Given valuations $\theta \in[T \rightarrow \mathbb{N}]$, $\delta \in[C \rightarrow \mathbb{C}]$, and $\mu \in[\Gamma \rightarrow(\mathbb{N} \rightarrow \mathbb{C})]$, we define a mapping $\langle\langle\cdot\rangle\rangle_{\theta, \delta, \mu}$ which associates with each color term a value in $\mathbb{C}$ :

$$
\begin{aligned}
\langle\langle z\rangle\rangle_{\theta, \delta, \mu} & =\delta(z) \\
\langle\langle\gamma(x)\rangle\rangle_{\theta, \delta, \mu} & =\mu(\gamma)(\theta(x)) \\
\left\langle\left\langle\omega\left(t_{1}, \ldots, t_{n}\right)\right\rangle\right\rangle_{\theta, \delta, \mu} & =\omega\left(\left\langle\left\langle t_{1}\right\rangle\right\rangle_{\theta, \delta, \mu}, \ldots,\left\langle\left\langle t_{n}\right\rangle\right\rangle_{\theta, \delta, \mu}\right)
\end{aligned}
$$

Then, we define inductively the satisfaction relation $\models_{\theta, \delta}$ between colored markings $\langle M, \mu\rangle$ and $\mathrm{CML}$ formulas as follows:

$$
\begin{aligned}
\langle M, \mu\rangle \models_{\theta, \delta} \xi\left(t_{1}, \ldots, t_{m}\right) \text { iff } \xi\left(\left\langle\left\langle t_{1}\right\rangle\right\rangle_{\theta, \delta, \mu}, \ldots,\left\langle\left\langle t_{m}\right\rangle\right\rangle_{\theta, \delta, \mu}\right) \\
\langle M, \mu\rangle \models_{\theta, \delta} p(x) \text { iff } M(\theta(x))=p \\
\langle M, \mu\rangle \models_{\theta, \delta} x=y \text { iff } \theta(x)=\theta(y) \\
\langle M, \mu\rangle \models_{\theta, \delta} \neg \varphi \text { iff }\langle M, \mu\rangle \models_{\theta, \delta} \varphi \\
\langle M, \mu\rangle \models_{\theta, \delta} \varphi_{1} \vee \varphi_{2} \text { iff }\langle M, \mu\rangle \models_{\theta, \delta} \varphi_{1} \text { or }\langle M, \mu\rangle \models_{\theta, \delta} \varphi_{2} \\
\langle M, \mu\rangle \models_{\theta, \delta} \exists x . \varphi \text { iff } \exists t \in \mathbb{T} .\langle M, \mu\rangle \models_{\theta[x \leftarrow t], \delta} \varphi \\
\langle M, \mu\rangle \models_{\theta, \delta} \exists z . \varphi \text { iff } \exists c \in \mathbb{C} .\langle M, \mu\rangle \models_{\theta, \delta[z \leftarrow c]} \varphi
\end{aligned}
$$

For every formula $\varphi$, we define $[[\varphi]]_{\theta, \delta}$ the be the set of markings $\langle M, \mu\rangle$ such that $\langle M, \mu\rangle \models_{\theta, \delta} \varphi$. A formula $\varphi$ is satisfiable iff there exist valuations $\theta$ and $\delta$ s.t. $[[\varphi]]_{\theta, \delta} \neq \emptyset$. 


\subsection{Syntactical Forms and Fragments}

Prenex normal form: A formula is in prenex normal form (PNF) if it is of the form

$$
Q_{1} y_{1} Q_{2} y_{2} \ldots Q_{m} y_{m} . \varphi
$$

where (1) $Q_{1}, \ldots, Q_{m}$ are (existential or universal) quantifiers, (2) $y_{1}, \ldots, y_{m}$ are variables in $T \cup C$, and $\varphi$ is a quantifier-free formula. It can be proved that for every formula $\varphi$ in $C M L$, there exists an equivalent formula $\varphi^{\prime}$ in prenex normal form.

Quantifier alternation hierarchy: We consider two families $\left\{\Sigma_{n}\right\}_{n \geq 0}$ and $\left\{\Pi_{n}\right\}_{n \geq 0}$ of fragments of $C M L$ defined according to the alternation depth of existential and universal quantifiers in their PNF:

- Let $\Sigma_{0}=\Pi_{0}$ be the set of formulas in PNF where all quantified variables are in $C$,

- For $n \geq 0$, let $\Sigma_{n+1}$ (resp. $\Pi_{n+1}$ ) be the set of formulas $Q y_{1} \ldots y_{m}$. $\varphi$ in PNF where $y_{1}, \ldots, y_{m} \in T \cup C, Q$ is the existential (resp. universal) quantifier $\exists$ (resp. $\forall$ ), and $\varphi$ is a formula in $\Pi_{n}\left(\right.$ resp. $\left.\Sigma_{n}\right)$.

It is easy to see that, for every $n \geq 0, \Sigma_{n}$ and $\Pi_{n}$ are closed under conjunction and disjunction, and that the negation of a $\Sigma_{n}$ formula is a $\Pi_{n}$ formula and vice versa. For every $n \geq 0$, let $B\left(\Sigma_{n}\right)$ denote the set of all boolean combinations of $\Sigma_{n}$ formulas. Clearly, $B\left(\Sigma_{n}\right)$ subsumes both $\Sigma_{n}$ and $\Pi_{n}$, and is included in both $\Sigma_{n+1}$ and $\Pi_{n+1}$.

Special form: The set of formulas in special form is given by the grammar:

$$
\varphi::=x=y\left|\xi\left(t_{1}, \ldots, t_{n}\right)\right| \neg \varphi|\varphi \vee \varphi| \exists z . \varphi \mid \exists x \in p . \varphi
$$

where $x, y \in T, z \in C, p \in \mathbb{P} \cup\{\perp\}, \xi \in \Xi$, and $t_{1}, \ldots, t_{n}$ are token color terms. It is not difficult to see that for every closed formula $\varphi$ in CML, there exists an equivalent formula $\varphi^{\prime}$ in special form. The transformation is based on the following fact: since variables are assumed to be quantified at most once in formulas, each formula $\exists x$. $\phi$ can be replaced by $\bigvee_{p \in \mathbb{P} \cup\{\perp\}} \exists x \in p$. $\phi_{x, p}$ where $\phi_{x, p}$ is obtained by substituting in $\phi$ each occurrence of $p(x)$ by true, and each occurrence of $q(x)$, with $p \neq q$, by false.

\section{Satisfiability Problem}

We investigate the decidability of the satisfiability problem of the logic $\operatorname{CML}(L)$, assuming that the underlying color logic $L$ has a decidable satisfiability problem.

Let us mention that in the case of a finite color domain, for instance for the domain of booleans with equality and usual operations, the logic CML is decidable. The result is a direct consequence of the decidability of the class of relational monadic formulae in first-order logic, also known as the Löwenheim class with equality [10].

Then, let us consider the case of infinite data domains. First, we prove that as soon as we consider natural numbers with ordering, the satisfiability problem of CML is undecidable already for the fragment $\Pi_{2}$. The proof is by a reduction of the halting problem of Turing machines. The idea is to encode a computation of a machine, seen as a sequence of tape configurations, using tokens with integer colors. Each token represents 
a cell in the tape of the machine at some computation step. Therefore the token has two integer colors, its position in the tape, and the position of its configuration in the computation (the corresponding computation step). The other informations such as the contents of the cell, the fact that a cell corresponds to the position of the head, and the control state, are encoded using a finite number of places. Then, using $\forall^{*} \exists^{*}$ formulas, it is possible to express that two consecutive configurations correspond indeed to a valid transition of the machine. Intuitively, this is possible because these formulas allow to relate each cell at some configuration to the corresponding cell at the next configuration.

Theorem 1. The satisfiability problem of the fragment $\Pi_{2}$ of $\mathrm{CML}(\mathrm{OL})$ is undecidable.

Nevertheless, we can prove the following generic decidability result for the fragment $\Sigma_{2}$ of our logic:

Theorem 2. Let L be a colored tokens logic. If the satisfiability problem of $L$ is decidable, then the fragment $\Sigma_{2}$ of $\mathrm{CML}(L)$ is decidable.

The idea of the proof is to reduce the satisfiability problem of $\Sigma_{2}$ to the satisfiability problem of $\Sigma_{0}$ formulas (which are formulas in the color logic $L$ ). We proceed as follows: we prove first that the fragment $\Sigma_{2}$ has the small model property, i.e., every satisfiable formula $\varphi$ in $\Sigma_{2}$ has a model of a bounded size (where the size is the number of tokens in each place). This bound corresponds actually to the number of existentially quantified token variables in the formula. Notice that this fact does not lead directly to an enumerative decision procedure for the satisfiability problem since the number of models of a bounded size is infinite in general (due to infinite color domains). Then, we use the fact that over a finite model, universal quantifications in $\varphi$ can be transformed into finite conjunctions, in order to build a formula $\widehat{\varphi}$ in $\Sigma_{1}$ which is satisfiable if and only if the original formula $\varphi$ is satisfiable. Actually, $\widehat{\varphi}$ defines precisely the upwardclosure of the set of markings defined by $\varphi$ (w.r.t. the inclusion ordering between sets of colored markings, extended to vectors of places). Finally, it can be shown that the $\Sigma_{1}$ formula $\widehat{\varphi}$ is satisfiable if and only if the $\Sigma_{0}$ obtained by transforming existential quantification over token into existential quantification over colors is decidable.

\section{Constrained Petri Nets}

Let $T$ be a set of token variables and $C$ be a set of color variables such that $T \cap C \neq 0$. A Constrained Petri Net (CPN) is a tuple $S=(\mathbb{P}, L, \Gamma, \Delta)$ where $\mathbb{P}$ is a finite set of places, $L=(\mathbb{C}, \Omega, \Xi)$ is a colored tokens logic, $\Gamma$ is a finite set of token coloring symbols, and $\Delta$ is a finite set of constrained transitions of the form:

$$
\vec{x} \in \vec{p} \hookrightarrow \vec{y} \in \vec{q}: \varphi(\vec{x}, \vec{y})
$$

where $\vec{x}=\left(x_{1}, \ldots, x_{n}\right) \in T^{n}, \vec{y}=\left(y_{1}, \ldots, y_{m}\right) \in T^{m}, \vec{p}=\left(p_{1}, \ldots, p_{n}\right) \in \mathbb{P}^{n}, \vec{q}=$ $\left(q_{1}, \ldots, q_{m}\right) \in \mathbb{P}^{m}$, and $\varphi(\vec{x}, \vec{y})$ is a $\operatorname{CML}(L, \Gamma, \mathbb{P})$ formula called the transition guard.

Given a fragment $\Theta$ of $C M L$, we denote by $C P N[\Theta]$ the class of CPN where all transition guards are formulas in the fragment $\Theta$. Due to the (un)decidability results of section 3 , we focus in the sequel on the classes $\operatorname{CPN}\left[\Sigma_{2}\right]$ and $\operatorname{CPN}\left[\Sigma_{1}\right]$. 
Configurations of CPN's are colored markings. Constrained transitions define transformation rules of these markings. Given a CPN $S$, we define a transition relation $\rightarrow_{S}$ between colored markings as follows: For every two colored markings $\langle M, \mu\rangle$ and $\left\langle M^{\prime}, \mu^{\prime}\right\rangle$, we have $\langle M, \mu\rangle \rightarrow_{S}\left\langle M^{\prime}, \mu^{\prime}\right\rangle$ iff there exists a constrained transition of the form (1), and there exist tokens $t_{1}, \ldots, t_{n}$ and $t_{1}^{\prime}, \ldots, t_{m}^{\prime}$ s.t. $\forall i, j \in\{1, \ldots, n\} . i \neq j \Rightarrow t_{i} \neq t_{j}$, and $\forall i, j \in\{1, \ldots, m\} . i \neq j \Rightarrow t_{i}^{\prime} \neq t_{j}^{\prime}$, and

1. $\forall i \in\{1, \ldots, n\} . M\left(t_{i}\right)=p_{i}$ and $M^{\prime}\left(t_{i}\right)=\perp$,

2. $\forall i \in\{1, \ldots, m\} . M\left(t_{i}^{\prime}\right)=\perp$ and $M^{\prime}\left(t_{i}^{\prime}\right)=q_{i}$,

3. $\forall t \in \mathbb{N}$, if $\forall i \in\{1, \ldots, n\} . t \neq t_{i}$ and $\forall j \in\{1, \ldots, m\} . t \neq t_{j}^{\prime}$, then $M(t)=M^{\prime}(t)$ and $\forall \gamma \in \Gamma \cdot \mu(\gamma)(t)=\mu^{\prime}(\gamma)(t)$

4. $\left\langle M, \mu \cup \mu^{\prime}\right\rangle=_{\theta, \delta_{\emptyset}} \varphi(\vec{x}, \vec{y})$, where $\theta \in[T \rightarrow \mathbb{N}]$ is a valuation of the token variables such that $\forall i \in\{1, \ldots, n\} . \theta\left(x_{i}\right)=t_{i}$ and $\forall j \in\{1, \ldots, m\} . \theta\left(y_{j}\right)=t_{j}^{\prime}, \delta_{\emptyset}$ is the empty domain valuation of color variables, and $\mu \cup \mu^{\prime}$ is such that: for every $\gamma \in \Gamma$, and every token $t \in \mathbb{T}$, if $t \in\left\{t_{1}, \ldots, t_{n}\right\}$ then $\mu \cup \mu^{\prime}(\gamma)(t)=\mu(\gamma)(t)$, if $t \in\left\{t_{1}^{\prime}, \ldots, t_{m}^{\prime}\right\}$ then $\mu \cup \mu^{\prime}(\gamma)(t)=\mu^{\prime}(\gamma)(t)$, and $\mu \cup \mu^{\prime}(\gamma)(t)=\mu(\gamma)(t)=\mu^{\prime}(\gamma)(t)$ otherwise.

Intuitively, the definition above says that firing a transition means that $n$ different tokens $t_{1}, \ldots, t_{n}$ are deleted from the places $p_{1}, \ldots, p_{n}(1)$, and $m$ new different tokens $t_{1}^{\prime}, \ldots, t_{m}^{\prime}$ are added to the places $q_{1}, \ldots, q_{m}(2)$, provided that the colors of all these (old and new) tokens satisfy the formula $\varphi$, which may also involve constraints on other tokens in the whole marking $M(4)$. Moreover, this operation does not modify the rest of the tokens (others than $t_{1}, \ldots, t_{n}$ and $t_{1}^{\prime}, \ldots, t_{m}^{\prime}$ ) in the marking (3).

Given a colored marking $\mathcal{M}$, let post ${ }_{S}(\mathcal{M})=\left\{\mathcal{M}^{\prime}: \mathcal{M} \rightarrow_{S} \mathcal{M}^{\prime}\right\}$ be the set of its immediate successors, and let $\operatorname{pre}_{S}(\mathcal{M})=\left\{\mathcal{M}^{\prime}: \mathcal{M}^{\prime} \rightarrow_{S} \mathcal{M}\right\}$ be the set of its immediate predecessors. These definitions can be generalized to sets of colored markings in the obvious way. Finally, for every set of colored markings $\mathbb{M}$, let $\widetilde{\operatorname{pre}_{S}}(\mathbb{M})=\overline{\operatorname{pre}_{S}(\overline{\mathbb{M}})}$, where $(\ulcorner)$ denotes complementation (w.r.t. the set of all colored markings).

\section{Modeling Power of CPN}

We show in this section how CPN can be used to model (unbounded) dynamic networks of parallel processes. We assume w.l.o.g. that all processes are identically defined. We consider that a process is defined by a finite control state machine supplied with variables and data structures ranging over potentially infinite domains (such as integer variables, reals, etc). Processes running in parallel can communicate and synchronize using various kinds of mechanisms (rendez-vous, shared variables, locks, etc). Moreover, they can dynamically spawn new (copies of) processes in the network.

Dynamic networks of processes: Let $\mathcal{L}$ be the set of control locations of each of the processes. (Remember that this set is the same for all processes.) We associate with each process control location $\ell \in \mathcal{L}$ a place. Then, each running process is represented by a token, and in every marking, a place contains precisely the tokens representing processes which are at the corresponding control location.

Assume for the moment that processes do not manipulate (infinite domain) data. Then, a basic action $\ell \longrightarrow \ell^{\prime}$ of a process moving its control from a location $\ell$ to another 
location $\ell^{\prime}$ is modeled by a transition: $x \in \ell \hookrightarrow y \in \ell^{\prime}:$ true. An action spawning a new process $\ell \stackrel{\operatorname{spawn}\left(\ell_{0}\right)}{\longrightarrow} \ell^{\prime}$ is modeled using a transition which creates a new token in the initial control location of the new process: $x \in \ell \hookrightarrow y_{1} \in \ell^{\prime}, y_{2} \in \ell_{0}:$ true.

Local variables: Consider now that each process has a vector of $n$ local variables $\vec{v}=\left(v_{1}, \ldots, v_{n}\right)$ over some (potentially infinite) data domain. Then, we consider a set of coloring symbols $\Gamma=\left\{\gamma_{1}, \ldots, \gamma_{n}\right\}$ associating with each token $n$ colors (in the considered data domain) corresponding to the values of the local variables: for each process, represented by a token $t$, for each local variable $v_{i}, \gamma_{i}(t)$ defines the value of $v_{i}$.

A process action $\ell \stackrel{\vec{v}:=\vec{f}(\vec{v})}{\longrightarrow} \ell^{\prime}$ which (in addition of changing the control location from $\ell$ to $\ell^{\prime}$ ) performs the assignment $\vec{v}:=\vec{f}(\vec{v})$, where $\vec{f}$ is a vector of expressions over the considered data domain, is modeled by the transition

$$
x \in \ell \hookrightarrow y \in \ell^{\prime}: \bigwedge_{i=1}^{n} \gamma_{i}(y)=f_{i}\left(\gamma_{1}(x), \ldots, \gamma_{n}(x)\right)
$$

For that, we use a token color logic which allows to express the effects of the actions. For instance, in the case of processes with integer variables and linear assignments, Presburger arithmetics (PA) can be used as colored tokens logic.

Global variables: Assume that processes share global variables $\vec{u}=\left\{u_{1}, \ldots, u_{m}\right\}$ (which are read and updated in a concurrent way). We associate with each global variable $u_{i}$ a place $g_{i}$ containing a single token $t_{i}$, and we associate with this token a color $\alpha\left(t_{i}\right)$ representing the value of $u_{i}$, where $\alpha \in \Gamma$ is a special coloring symbol. Then, a process action $\ell \stackrel{\vec{u}:=\vec{f}(\vec{u}, \vec{v})}{\longrightarrow} \ell^{\prime}$ (assigning to global variables values depending on both global variables and local variables of the process) is modeled by the transition:

$$
\begin{gathered}
x \in \ell, x_{1} \in g_{1}, \ldots, x_{m} \in g_{m} \hookrightarrow y \in \ell^{\prime}, y_{1} \in g_{1}, \ldots, y_{m} \in g_{m}: \\
\left(\bigwedge_{i=1}^{n} \gamma_{i}(y)=\gamma_{i}(x)\right) \wedge \bigwedge_{i=1}^{m} \alpha\left(y_{i}\right)=f_{i}\left(\alpha\left(x_{1}\right), \ldots, \alpha\left(x_{m}\right), \gamma_{1}(x), \ldots, \gamma_{n}(x)\right)
\end{gathered}
$$

In the modeling above, we consider that the execution of the process action is atomic. When assignments are not atomic, we must transform each of assignment action into a sequence of atomic operations: read first the global variables and assign their values to local variables, then compute locally the new values to be assigned to global variables, and finally assign these values to global variables.

Rendez-vous synchronization: Synchronization between a finite number of processes can be modeled as in Petri nets. CPNs allow in addition to put constraints on the colors (data) of the involved processes.

Priorities: Various notion of priorities, such as priorities between different classes of processes (defined by properties of their colors), or priorities between different actions, can be modeled in CPNs. This can be done by imposing in transition guards that transitions (performed by processes or corresponding to actions) of higher priority are not 
enabled. These constraints can be expressed using $\Pi_{1}$ formulas. In particular, checking that a place $p$ is empty can be expressed by $\forall x \in p$. false. (Which shows that as soon as universally quantified formulas are allowed in guards, our models are as powerful as Turing machines, even for color logics over finite domains.)

Process identities: It is possible to associate with each newly created process an identity defined by an integer number. For that, we consider a special coloring symbol $I d \in \Gamma$ associating to each token the identity of the process it represents. To ensure that different processes have different identities, we express in the guard of every transition which creates a process (i.e., adds a token to the place corresponding to its initial control location) the fact that the identity of this process does not exist already among tokens in places corresponding to control locations. This can easily be done using a universally quantified $\left(\Pi_{1}\right)$ formula. Therefore, a spawn action $\ell \stackrel{\operatorname{spawn}\left(\ell_{0}\right)}{\longrightarrow} \ell^{\prime}$ is modeled by:

$$
\begin{gathered}
x \in \ell \hookrightarrow y_{1} \in \ell^{\prime}, y_{2} \in \ell_{0}: \\
I d(x)=I d\left(y_{1}\right) \wedge\left(\bigwedge_{i=1}^{n} \gamma_{i}\left(y_{1}\right)=\gamma_{i}(x)\right) \wedge \bigwedge_{\text {loc } \in \mathcal{L}} \forall t \in \text { loc. } \neg\left(\operatorname{Id}\left(y_{2}\right)=\operatorname{Id}(t)\right)
\end{gathered}
$$

and the modeling of other actions (such as local/global variables assignments) can be modified accordingly in order to propagate the process identity through the transition. Notice that process identities are different from token values. Indeed, in some cases (e.g., for modeling value passing as described below), we may use different tokens (at some special places representing buffers for instance) corresponding to the same $I d$.

Locks: Locks can be simply modeled using global variables storing the identity of the owner process, or a special value (e.g. - 1) if it is free. A process who acquires the lock must check if it is free, and then write his identity:

$$
x_{1} \in \ell, x_{2} \in \text { lock } \hookrightarrow y_{1} \in \ell^{\prime}, y_{2} \in \text { lock }: \alpha\left(x_{2}\right)=-1 \wedge \alpha\left(y_{2}\right)=\operatorname{Id}\left(x_{1}\right) \wedge \ldots
$$

To release the lock, a process assigns -1 to the lock, which can be modeled in a similar way. Other kinds of locks, such as reader-writer locks, can also be modeled in our framework (see [14]). The modeling of such locks when the number of readers and writers can be arbitrarily large requires the use of universal quantification in guards.

Value passing, return values: Processes may pass/wait for values to/from other processes with specific identities. They can use for that shared arrays of data indexed by process identities. Such an array $A$ can be modeled in our framework using a special place containing for each process a token. Initially, this place is empty, and whenever a new process is created, a token with the same identity is added to this place. Then, to model that a process read/write on $A[i]$, we use a transition which takes from the place associated with $A$ the token with $I d$ equal to $i$, read/modifies the value attached with this token, and put the token again in the same place. For instance, an assignment action $\ell \stackrel{A[k]:=e}{\longrightarrow} \ell^{\prime}$ executed by some process is modeled by the transition:

$$
x_{1} \in \ell, x_{2} \in A \hookrightarrow y_{1} \in \ell^{\prime}, y_{2} \in A:
$$




$$
I d\left(x_{1}\right)=I d\left(y_{1}\right) \wedge\left(\bigwedge_{i=1}^{n} \gamma_{i}\left(x_{1}\right)=\gamma_{i}\left(y_{1}\right)\right) \wedge I d\left(x_{2}\right)=k \wedge \alpha\left(y_{2}\right)=e \wedge I d\left(y_{2}\right)=\operatorname{Id}\left(x_{2}\right)
$$

Notice that, while it is possible to model using CPNs systems manipulating parametricsize arrays (using multisets of tokens with integer colors), we cannot express in the decidable fragment $\Sigma_{2}$ of CML the fact that a multiset indeed encodes an array of elements indexed by integers in some given interval. The reason is that, while we can express in $\Pi_{1}$ the fact that each token has a unique color in the interval, we need to use $\Pi_{2}$ formulas to say that for each color in the interval there exists a token with that color. Nevertheless, for the verification of safety properties and checking invariants, it is not necessary to require the latter property.

\section{Computing post and pre Images}

We prove hereafter closure properties of CML fragments under the computation of immediate successors and predecessors for CPNs. The main result of this section is:

Theorem 3. Let $S$ be a CPN $\left[\Sigma_{n}\right]$, for $n \in\{1,2\}$. Then, for every closed formula $\varphi$ in the fragment $\Sigma_{n}$ of $\mathrm{CML}$, it is possible to construct two closed formulas $\varphi_{\text {post }}$ and $\varphi_{\text {pre }}$ in the same fragment $\Sigma_{n}$ such that $\left[\left[\varphi_{\text {post }} \rrbracket\right]=\operatorname{post}_{S}([\varphi]]\right)$ and $\left[\left[\varphi_{\text {pre }}\right]\right]=\operatorname{pre}_{S}([\varphi])$.

We give hereafter a sketch of the proof. Let $\varphi$ be a closed formula, and let $\tau$ be a transition $\vec{x} \in \vec{p} \hookrightarrow \vec{y} \in \vec{q}: \psi$ of the system $S$. W.1.o.g., we suppose that $\varphi$ and $\psi$ are in special form. We define hereafter the formulas $\varphi_{\text {post }}$ and $\varphi_{\text {pre }}$ for this single transition. The generalization to the set of all transitions is straightforward.

The construction of the formulas $\varphi_{\text {post }}$ and $\varphi_{\text {pre }}$ is not trivial because our logic does not allow to use quantification over places and color mappings (associated with coloring symbols). Intuitively, the idea is to express first the effect of deleting/adding tokens, and then composing these operations to compute the effect of a transition.

Let us introduce two transformations $\ominus$ and $\oplus$ corresponding to deletion and creation of tokens. These operations are inductively defined on the structure of special form formulas in Table 1

The operation $\ominus$ is parameterized by a vector $\vec{z}$ of token variables to be deleted, a mapping loc associating with token variables in $\vec{z}$ the places from which they will be deleted, and a mapping col associating with each coloring symbol in $\Gamma$ and each token variable in $\vec{z}$ a fresh color variable in $C$. Intuitively, $\ominus$ projects a formula on all variables which are not in $\vec{z}$. Rule $\ominus_{1}$ substitutes in a color formula $\xi(\vec{t})$ all occurences of colored tokens in $\vec{z}$ by fresh color variables given by the mapping col. A formula $x=y$ is unchanged by the application of $\ominus$ is the token variables $x$ and $y$ are not in $\vec{z}$; otherwise, rule $\ominus_{2}$ replaces $x=y$ by true if it is trivially true (i.e., we have the same variable in both sides of the equality) or by false if $x$ or $y$ is in $\vec{z}$. Indeed, each token variable in $\vec{z}$ represents (by the semantics of CPN) a different token, and since this token is deleted by the transition rule, it cannot appear in the reached configuration. Rules $\ominus_{3}$ and $\ominus_{4}$ are straightforward. Finally, rule $\ominus_{5}$ does a case splitting according to the fact whether a deleted token is precisely the one referenced by the existential token quantification or not. 
The operation $\oplus$ is parameterized by a vector $\vec{z}$ of token variables to be added and a mapping loc associating with each variable in $z \in \vec{z}$ a place (in which it will be added). Intuitively, $\oplus$ transforms a formula taking into account that the added tokens by the transition were not present in the previous configuration (and therefore not constrained by the original formula describing the configuration before the transition). Then, the application of $\oplus$ has no effect on color formulas $\xi(\vec{t})$ (rule $\oplus_{1}$ ). When equality of tokens is tested, rule $\oplus_{2}$ takes into account that all added tokens are distinct and different from the existing tokens. For token quantification, rule $\oplus_{5}$ says that quantified tokens of the previous configuration cannot be equal to the added tokens.

Then, we define $\varphi_{\text {post }}$ to be the formula:

$$
\exists \vec{y} \in \vec{q} \cdot \exists \vec{c} \cdot((\varphi \wedge \psi) \ominus(\vec{x}, \vec{x} \mapsto \vec{p}, \Gamma \mapsto(\vec{x} \mapsto \vec{c}))) \oplus(\vec{y}, \vec{y} \mapsto \vec{q})
$$

In the formula above, we first delete the tokens corresponding to $\vec{x}$ from the current configuration $\varphi$ intersected with the guard of the rule $\psi$. Then, we add tokens corresponding to $\vec{y}$. Finally, we close the formula by quantifying existentially the color variables and the token variables corresponding to the added tokens.

Similarly, we define $\varphi_{\text {pre }}$ to be the formula:

$$
\exists \vec{x} \in \vec{p} \cdot \exists \vec{c} \cdot((\varphi \oplus(\vec{x}, \vec{x} \mapsto \vec{p})) \wedge \psi) \ominus(\vec{y}, \vec{y} \mapsto \vec{q}, \Gamma \mapsto(\vec{y} \mapsto \vec{c}))
$$

For predecessor computation, we add to the current configuration the tokens represented by the left hand side of the rule $\vec{x}$ in order to obtain a configuration on which the guard $\psi$ can be applied. Then, we remove the tokens added by the rule using token variables $\vec{y}$. Finally, we close the formula by quantifying existentially the color variables and the token variables corresponding to the added tokens. It is easy to see that if $\varphi$ and $\psi$ are in a fragment $\Sigma_{n}$, for any $n \geq 1$, then both of the formulas $\varphi_{\text {post }}$ and $\varphi_{\text {pre }}$ are also in the same fragment $\Sigma_{n}$.

Corollary 1. Let $S$ be a CPN $\left[\Sigma_{1}\right]$. Then, for every formula $\varphi$ in $\Pi_{1}$, it is possible to construct a formula $\varphi_{\widetilde{\text { pre }}}$ also in $\Pi_{1}$ s.t. $\left[\left[\varphi_{\widetilde{\text { pre }}}\right]\right]=\widetilde{\text { pre }_{S}}([[\varphi]])$.

\section{Applications in Verification}

We show in this section how to use the results of the previous section to perform various kinds of analysis. Let us fix for the rest of the section a colored tokens logic $L$ with a decidable satisfiability problem, and a CPN $S$ defined over $L$ and the logic $\operatorname{CML}(L)$.

\subsection{Pre-post Condition Reasoning}

Given a transition $\tau$ in $S$ and given two formulas $\varphi$ and $\varphi^{\prime},\left\langle\varphi, \tau, \varphi^{\prime}\right\rangle$ is a Hoare triple if whenever the condition $\varphi$ holds, the condition $\varphi^{\prime}$ holds after the execution of $\tau$. In other words, we must have post $([[\varphi]]) \subseteq\left[\left[\varphi^{\prime}\right]\right]$, or equivalently that $\operatorname{post}_{\tau}([[\varphi]]) \cap\left[\left[\neg \varphi^{\prime}\right]\right]=\emptyset$. Then, by Theorem 3 and Theorem 2 we deduce the following:

Theorem 4. If $S$ is a $\operatorname{CPN}\left[\Sigma_{2}\right]$, then the problem whether $\left\langle\varphi, \tau, \varphi^{\prime}\right\rangle$ is a Hoare triple is decidable for every transition $\tau$ of $S$, every formula $\varphi \in \Sigma_{2}$, and every formula $\varphi^{\prime} \in \Pi_{2}$. 
Table 1. Definition of the $\oplus$ and $\ominus$ operators

$$
\begin{aligned}
& \ominus_{1}: \quad \xi(\vec{t}) \ominus(\vec{z}, \text { loc, col })=\xi(\vec{t})[\operatorname{col}(\gamma)(z) / \gamma(z)]_{\gamma \in \Gamma, z \in \vec{z}} \\
& \ominus_{2}: \quad(x=y) \ominus(\vec{z}, \text { loc }, \text { col })= \begin{cases}x=y & \text { if } x, y \notin \vec{z} \\
\text { true } & \text { if } x \equiv y \\
\text { false } & \text { otherwise }\end{cases} \\
& \ominus_{3}: \quad(\neg \varphi) \ominus(\vec{z}, \text { loc }, \text { col })=\neg(\varphi \ominus(\vec{z}, \text { loc }, \operatorname{col})) \\
& \ominus_{4}: \quad\left(\varphi_{1} \vee \varphi_{2}\right) \ominus(\vec{z}, \text { loc }, \text { col })=\left(\varphi_{1} \ominus(\vec{z}, \text { loc }, \text { col })\right) \vee\left(\varphi_{2} \ominus(\vec{z}, \text { loc }, \text { col })\right) \\
& \ominus_{5}:(\exists x \in p . \varphi) \ominus(\vec{z}, \text { loc }, \operatorname{col})=\exists x \in p .(\varphi \ominus(\vec{z}, \text { loc }, \operatorname{col})) \vee \\
& \bigvee_{z \in \vec{z}: \operatorname{loc}(z)=p}(\varphi[z / x]) \ominus(\vec{z}, 1 \circ c, \operatorname{col}) \\
& \oplus_{1}: \quad \xi(\vec{t}) \oplus(\vec{z}, \text { loc })=\xi(\vec{t}) \\
& \oplus_{2}: \quad(x=y) \oplus(\vec{z}, \text { loc })= \begin{cases}x=y & \text { if } x, y \notin \vec{z} \\
\text { true } & \text { if } x \equiv y \\
\text { false } & \text { otherwise }\end{cases} \\
& \oplus_{3}: \quad(\neg \varphi) \oplus(\vec{z}, \text { loc })=\neg(\varphi \oplus(\vec{z}, \text { loc })) \\
& \oplus_{4}: \quad\left(\varphi_{1} \vee \varphi_{2}\right) \oplus(\vec{z}, \text { loc })=\left(\varphi_{1} \oplus(\vec{z}, \text { loc })\right) \vee\left(\varphi_{2} \oplus(\vec{z}, \text { loc })\right) \\
& \oplus_{5}: \quad(\exists x \in p \cdot \varphi) \oplus(\vec{z}, \text { loc })=\exists x \in p .(\varphi \oplus(\vec{z}, \text { loc })) \wedge \bigwedge_{z \in \vec{z}: \operatorname{loc}(z)=p} \neg(x=z)
\end{aligned}
$$

\subsection{Bounded Reachability Analysis}

An instance of the bounded reachability analysis problem is a triple (Init,Target, $k$ ) where Init and Target are two sets of configurations, and $k$ is a positive integer. The problem consists in deciding whether there exists a computation of length at most $k$ which starts from some configuration in Init and reaches a configuration in Target. In other words, the problem consists in deciding whether Target $\cap \bigcup_{0<i<k} \operatorname{post}_{S}^{i}($ Init $) \neq \emptyset$, or equivalently whether Init $\cap \bigcup_{0 \leq i \leq k} \operatorname{pre}_{S}^{i}($ Target $) \neq 0$. The following result is a direct consequence of Theorem 3 and Theorem 2 .

Theorem 5. If $S$ is a $\operatorname{CPN}\left[\Sigma_{2}\right]$, then, for every $k \in \mathbb{N}$, and for every two formulas $\varphi_{I}, \varphi_{T} \in \Sigma_{2}$, the bounded reachability problem $\left(\left[\left[\varphi_{I}\right]\right],\left[\left[\varphi_{T}\right]\right], k\right)$ is decidable.

\subsection{Checking Invariance Properties}

An instance of the invariance checking problem is given by a pair of sets of configurations (colored markings) (Init,Inv), and consists in deciding whether starting from any configuration in Init, every computation of $S$ can only visit configurations in Inv, i.e., $\bigcup_{k>0} \operatorname{post}_{S}^{k}($ Init $) \subseteq$ Inv. This problem is of course undecidable in general. However, a deductive approach using inductive invariants (provided by the user) can be adopted. We show that our results allow to automatize the steps of this approach.

A set of configurations $\mathbb{M}$ is an inductive invariant if $\operatorname{post}_{S}(\mathbb{M}) \subseteq \mathbb{M}$, or equivalently, if $\mathbb{M} \subseteq \widetilde{\operatorname{pre}_{S}}(\mathbb{M})$. By Theorem 3 and Theorem 2] we have:

Theorem 6. If $S$ is a CPN $\left[\Sigma_{2}\right]$, then for every formula $\varphi$ in $B\left(\Sigma_{1}\right)$, the problem of checking whether $\varphi$ defines an inductive invariant is decidable. 
The deductive approach for establishing an invariance property considers the inductive invariance checking problem given by a triple (Init, Inv, Aux) of sets of configurations, and which consists in deciding whether (1) Init $\subseteq A u x$, (2) Aux $\subseteq$ Inv, and (3) Aux is an inductive invariant. Indeed, a (sound and) complete rule for solving an invariance checking problem (Init, Inv) consists in finding a set of configurations Aux allowing to solve the inductive invariance checking problem (Init, Inv, Aux). The following result follows directly from Theorem 3 , Theorem 2 , and the previous theorem.

Theorem 7. If $S$ is a $\mathrm{CPN}\left[\Sigma_{2}\right]$, then the inductive invariance checking problem is decidable for every instance $\left(\left[\left[\varphi_{\text {Init }}\right]\right],[[\varphi]],\left[\left[\varphi^{\prime}\right]\right]\right)$ where $\varphi_{\text {Init }} \in \Sigma_{2}$, and $\varphi, \varphi^{\prime} \in B\left(\Sigma_{1}\right)$.

Of course, the difficult part in applying the deductive approach is to find useful auxiliary inductive invariants. One approach to tackle this problem is to try to compute the largest inductive invariant included in Inv which is the set $\bigcap_{k \geq 0} \widetilde{\operatorname{pre}}_{S}^{k}(\operatorname{Inv})$. Therefore, a method to derive auxiliary inductive invariants is to try iteratively the sets $\operatorname{Inv}, \operatorname{Inv} \cap \widetilde{\mathrm{pre}}_{S}(\operatorname{Inv})$, $I n v \cap \widetilde{\operatorname{pre}}_{S}(I n v) \cap \widetilde{\operatorname{pre}}_{S}^{2}(I n v)$, etc. In many practical cases, only few strengthening steps are needed to find an inductive invariant. (Indeed, the user is able in general to provide accurate invariant assertions for each control point of his system.) The result below implies that the steps of this iterative strengthening method can be automatized when CPN $\left[\Sigma_{1}\right]$ models and $\Pi_{1}$ invariants are considered. This result is a direct consequence of Corollary 1 .

Theorem 8. If $S$ is a $\mathrm{CPN}\left[\Sigma_{1}\right]$, then for every formula $\varphi$ in $\Pi_{1}$ and every positive integer $k$, it is possible to construct a formula in $\Pi_{1}$ defining the set $\bigcap_{0 \leq i \leq k} \widetilde{\operatorname{pre}}_{S}^{i}([\varphi \varphi])$.

We show in the full paper the applicability of our framework on a nontrivial example. We present the verification of a Reader-Writer lock for an unbounded number of processes using the inductive invariant checking approach. This example has been considered in [28] for a fixed number of processes.

\section{Conclusion}

We have presented a framework for reasoning about dynamic/parametric networks of processes manipulating data over infinite domains. We have provided generic models for these systems and a logic allowing to specify their configurations, both being parametrized by a logic on the considered data domain. We have identified a fragment of this logic having a decidable satisfiability problem and which is closed under post and pre image computation, and we have shown the application of these results in verification.

The complexity of the decision procedure and of the post/pre computation is exponential in the size of the formula, and more precisely in the number of quantified variables. However, formulas which appear in the analysis of systems such as parametrized/dynamic networks (such as assertions expressing invariants at each particular control location) are naturally in special form (see definition in Section 2.3) where each token variable is bound to a unique place (this allows to avoid the case splitting according to all possible mappings between token variables and places), and moreover, new token variables introduced by post/pre computations are of a fixed small number 
(the number of synchronized processes by the considered transition which is in general equal to two). These facts reduce significantly the complexity in practice.

Our framework allows to deal in a uniform way with all classes of systems manipulating infinite data domains with a decidable first-order theory. In this paper, we have considered instantiations of this framework based on logics over integers or reals (which allows to consider systems with numerical variables). Different data domains can be considered in order to deal with other classes of systems such as multithreaded programs where each process (thread) has an unbounded stack (due to procedure calls). We will address in more details the issue of applying our framework to the verification of multithreaded programs in a forthcoming paper. Our future work includes also the extension of our framework to other classes of systems and features such as dynamic networks of timed processes, networks of processes with broadcast communication, interruptions and exception handling, etc.

\section{References}

1. P. A. Abdulla, K. Cerans, B. Jonsson, and Y.-K. Tsay. General decidability theorems for infinite-state systems. In Proc. of LICS'96, pages 313-321, 1996.

2. P. A. Abdulla and G. Delzanno. On the Coverability Problem for Constrained Multiset Rewriting. In Proc. of AVIS'06, Satellite workshop of ETAPS'06, Vienna, Austria, 2006.

3. P. A. Abdulla, B. Jonsson, M. Nilsson, and M. Saksena. A Survey of Regular Model Checking. In Proc. of CONCUR'04, volume 3170 of LNCS. Springer, 2004.

4. Parosh Aziz Abdulla and Bengt Jonsson. Verifying networks of timed processes (extended abstract). In Bernhard Steffen, editor, Proc. of TACAS'98, volume 1384 of LNCS, pages 298-312. Springer, 1998.

5. A. Annichini, E. Asarin, and A. Bouajjani. Symbolic techniques for parametric reasoning about counter and clock systems. In Proc. of CAV'O0. LNCS 1855, 2000.

6. T. Arons, A. Pnueli, S. Ruah, J. Xu, and L.D. Zuck. Parameterized Verification with Automatically Computed Inductive Assertions. In Proc. of CAV'01, volume 2102 of LNCS. Springer, 2001.

7. Bernard Boigelot. Symbolic Methods for Exploring Infinite State Space. PhD thesis, Faculté des Sciences, Université de Liège, volume 189, 1999.

8. M. Bojanczyk, C. David, A. Muscholl, Th. Schwentick, and L. Segoufin. Two-variable logic on data trees and XML reasoning. In Proc. of PODS'06. ACM, 2006.

9. M. Bojanczyk, A. Muscholl, Th. Schwentick, L. Segoufin, and C. David. Two-variable logic on words with data. In Proc. of LICS'06. IEEE, 2006.

10. E. Börger, E. Grädel, and Y. Gurevich. The Classical Decision Problem. Perspectives of Mathematical Logic. Springer-Verlag, 1997. Second printing (Universitext) 2001.

11. A. Bouajjani, J. Esparza, and O. Maler. Reachability analysis of pushdown automata: Application to model-checking. In Proc. of CONCUR'97, volume 1243 of LNCS, pages 135-150. Springer, 1997.

12. A. Bouajjani, P. Habermehl, and T. Vojnar. Abstract Regular Model Checking. In Proc. of CAV'04, volume 3114 of $L N C S$. Springer, 2004.

13. A. Bouajjani, B. Jonsson, M. Nilsson, and T. Touili. Regular Model Checking. In Proc. of CAV'O0, volume 1855 of LNCS. Springer, 2000.

14. A. Bouajjani, Y. Jurski, and M. Sighireanu. A generic framework for reasoning about dynamic networks of infinite-state processes. Technical Report 2007-01, LIAFA lab, January 2007. Available at http://www.liafa.jussieu.fr/ abou/publis.html. 
15. A. Bouajjani, M. Müller-Olm, and T. Touili. Regular symbolic analysis of dynamic networks of pushdown systems. In Proc. of CONCUR'05, volume 3653 of LNCS. Springer, 2005.

16. A. Bouajjani and T. Touili. On computing reachability sets of process rewrite systems. In Proc. of RTA'05, volume 3467 of LNCS. Springer, 2005.

17. Ahmed Bouajjani. Languages, Rewriting systems, and Verification of Infinte-State Systems. In Proc. of ICALP'01, volume 2076 of LNCS. Springer Pub., 2001.

18. M. Bozzano and G. Delzanno. Beyond Parameterized Verification. In Proc. of TACAS'O2, volume 2280 of $L N C S$, Grenoble, France, 2002. Springer Pub.

19. A. R. Bradley, Z. Manna, and H. B. Sipma. What's decidable about arrays ? In Proc. of VMCAI'O6, volume 3855 of LNCS. Springer, 2006.

20. E. M. Clarke, O. Grumberg, and S. Jha. Verifying parameterized networks. TOPLAS, 19(5), 1997.

21. G. Delzanno. An assertional language for the verification of systems parametric in several dimensions. Electr. Notes Theor. Comput. Sci., 50(4), 2001.

22. G. Delzanno, J.-F. Raskin, and L. Van Begin. Towards the automated verification of multithreaded java programs. In TACAS, volume 2280 of LNCS, pages 173-187. Springer, 2002.

23. S. Demri and R. Lazic. LTL with the freeze quantifier and register automata. In Proc. of LICS'06. IEEE, 2006.

24. E. A. Emerson and K. S. Namjoshi. On model checking for non-deterministic infinite-state systems. In LICS'98. IEEE, 1998.

25. J. Esparza, A. Finkel, and R. Mayr. On the verification of broadcast protocols. In Proceedings of LICS '99, pages 352-359. IEEE Computer Society, 1999.

26. A. Finkel and J. Leroux. How to compose presburger-accelerations: Applications to broadcast protocols. In Proc. of FST\&TCS'02, volume 2556 of LNCS. Springer, 2002.

27. A. Finkel and $\mathrm{Ph}$. Schnoebelen. Well-structured transition systems everywhere! Theor. Comput. Sci., 256(1-2):63-92, 2001.

28. C. Flanagan, S.N. Freund, and S. Qadeer. Thread-modular verification for shared-memory programs. In Proc. of ESOP'O2, pages 262-277. LNCS 2305, 2002.

29. S. M. German and P. A. Sistla. Reasoning about systems with many processes. JACM, 39(3), 1992.

30. P. Wolper and B. Boigelot. Verifying systems with infinite but regular state spaces. In Proc. of CAV'98, volume 1427 of LNCS. Springer, 1998.

31. P. Wolper and V. Lovinfosse. Verifying properties of large sets of processes with network invariants. In Proc. Intern. Workshop on Automatic Verification Methods for Finite State Systems. LNCS 407, 1989. 